\title{
Enhanced super-radiance from energy-modulated short electron bunch free-electron lasers
}

\author{
Yu. Lurie* and Y. Pinhasi \\ Ariel University Center of Samaria, Ariel, Israel \\ (Received 17 April 2007; published 31 August 2007)
}

\begin{abstract}
In this paper we show that free-electron laser (FEL) super-radiant (SR) emission can be greatly enhanced by means of energy modulation of the radiating electron bunch. In FELs, super-radiant emission from short electron bunches is strongly reduced as the temporal duration of the bunch is increased. This prevents the realization of SR FELs operating at submillimeter wavelengths. We analyze the performance of a SR FEL source operating in the THz regime, which is driven by an energy-modulated short duration electron bunch. We carried out analytical evaluations and numerical simulations utilizing a spacefrequency $3 \mathrm{D}$ model. These show that a remarkable improvement in super-radiant emission intensity can be achieved by the use of appropriate linear modulation (chirp) of the beam energy within the bunch. Possible limitations in applying this method are discussed, as well as the spectral purity of the emitted radiation.
\end{abstract}

DOI: 10.1103/PhysRevSTAB.10.080703

PACS numbers: 41.60.Cr, 52.59.Rz

\section{INTRODUCTION}

Electron devices such as microwave tubes and freeelectron lasers (FELs) utilize distributed interaction between a drive electron beam and an electromagnetic field. In free-electron lasers, accelerated electrons pass through an undulator (wiggler) and radiate electromagnetic waves inside a cavity, as illustrated schematically in Fig. 1. Each electron in the beam emits partially coherent radiation, called undulator synchrotron radiation [1]. Random electron distribution in the beam causes fluctuations in current density, identified as shot noise in the beam current [2-5]. The total radiated electromagnetic field is obtained by summation of the contributions of radiation of individual electrons, resulting in a spontaneous emission [6-14].

If the electron beam is premodulated and electron bunches shorter than the oscillation period of the emitted radiation [15-19] are produced, the wave packets of the undulator synchrotron radiation emitted by each of the electrons in the beam add up in the phase, generating enhanced super-radiant (SR) emission [20]. Relations for power spectral densities of spontaneous and super-radiant emissions were developed in $[21,22]$, showing that superradiant emission is highly dependent on the bunch duration. Radiation is greatly reduced if bunch duration is increased due to the reduction of coherence between wave packets emitted by different electrons in the bunch. For example, for Gaussian density distribution of electrons in a bunch, the reduction of SR emission at a central radiation frequency $f_{s}$ was shown to be [21]

$$
\mathrm{SNR} \equiv \frac{d P_{q}^{s r}(z) / d f}{d P_{q}^{s p}(z) / d f}=\bar{k} \cdot e^{-\left(\pi f_{s} T_{b}\right)^{2}}
$$

(here $\bar{k}$ is the number of electrons in the bunch and $T_{b}$ is the bunch duration).

\footnotetext{
*ylurie@yosh.ac.il
}

Consequently, ultrashort electron bunches with a time duration $T_{b} \ll 1 / f_{s}$ should be used in order to generate SR emission at short wavelengths. State-of-the-art experimental techniques do not enable production of such short electron pulses; this prevents generation of SR emission at terahertz frequencies or higher. To enable SR emission at $\mathrm{THz}$ frequencies, we consider a FEL configuration utilizing controlled energy modulation of short $\left(T_{b} \simeq 1 / f_{s}\right)$ electron bunches. We shall show that appropriate energy modulation may provide bunch density compression along the interaction region, reducing destructive interference of undulator synchrotron radiation wave packets emitted by electrons from different parts of an electron bunch and then resulting in enhanced SR emission. Enhancement of coherent emission from radio-frequency modulated electron beams was demonstrated by use of energy-phase correlation in the beam [23]. Increase of Cherenkov SR emission in a dielectrically loaded waveguide by means of optimization of electron bunch energy profile was investigated in [24] (pulses much longer than the radiation period were considered in this work).

In the present work, the effect of energy modulation of wiggling short electron bunches on SR emission is studied in the framework of a space-frequency 3D analytical model

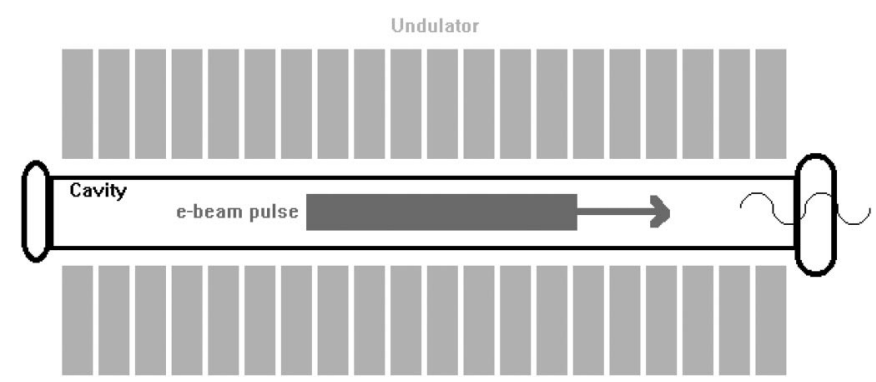

FIG. 1. Schematic illustration of a pulsed beam free-electron laser. 
and in numerical simulations carried out using the WB3D code [25]. The results obtained using these models are discussed; the spectral purity of the enhanced radiation is also considered.

\section{SPACE-FREQUENCY 3D APPROACH}

The total electromagnetic field excited by a wiggling electron bunch can be described in the frequency domain in terms of an expansion over the transverse eigenmodes of the medium (free-space or waveguide) in which the radiation is excited and propagates [26]:

$$
\vec{E}(\vec{r}, t)=\sum_{q} \operatorname{Re}\left[\int_{0}^{\infty} \tilde{C}_{q}(z, f) \tilde{\mathcal{E}}_{q}(x, y) e^{+j k_{z q}(f) z} d f\right],
$$

where $\tilde{\mathcal{E}}_{q}(x, y)$ is the transverse profile of mode $q$ (a Hermite-Gaussian free-space mode or a waveguide mode) and $k_{z q}(f)$ is its wave number. Although the mode summation presentation equation (2) is not valid for farfield free-space propagation, it is applicable to most electron devices in which the interaction takes place within a Rayleigh length of the Hermite-Gaussian modes, where diffraction is small. $\tilde{C}_{q}(z, f)$ is the $q$ th mode amplitude, found from the excitation equation [25,26]:

$$
\begin{aligned}
\frac{d}{d z} C_{ \pm q}(z, f)= & \mp \frac{1}{2 \mathcal{N}{ }_{q}(f)} e^{ \pm j k_{z q} z} \\
& \times \iint\left[\tilde{\mathbf{J}}_{\perp}(\mathbf{r}, f)+\hat{\mathbf{z}} \tilde{J}_{z}(\mathbf{r}, f)\right] \cdot \tilde{\mathcal{E}}_{ \pm q}^{*}(x, y) d x d y .
\end{aligned}
$$

Here

$$
\mathcal{N}_{q}(f)=\iint\left[\tilde{\mathcal{E}}_{q \perp} \times \tilde{\mathcal{H}}_{q \perp}^{*}\right] \cdot \hat{\mathbf{z}} d x d y
$$

is the power normalization of mode $q ; x, y$ are the transverse coordinates and $z$ is the longitudinal axis of propagation. The frequency-domain current density $\tilde{\mathbf{J}}(\mathbf{r}, f)$ of the drive beam can be expressed as a summation over a number of charged, pointlike electron "clusters" or "particles":

$$
\begin{aligned}
\mathbf{J}(\mathbf{r}, t) & =-\sum_{i} Q_{i} \mathbf{v}_{i} \delta\left(x-x_{i}\right) \delta\left(y-y_{i}\right) \delta\left[z-z_{i}(t)\right] \\
& =-\sum_{i} Q_{i}\left(\frac{\mathbf{v}_{i}}{v_{z_{i}}}\right) \delta\left(x-x_{i}\right) \delta\left(y-y_{i}\right) \delta\left[t-t_{i}(z)\right] .
\end{aligned}
$$

Here $Q_{i}$ is the charge of the $i$ th macroparticle in the simulation and $t_{i}(z)$ is the time it takes for a particle to arrive at a position $z$. The state and dynamics of the $i$ 's particle are described by a six-component vector, which consists of the particle's position coordinates $\mathbf{r}_{i}=$ $\left(x_{i}, y_{i}, z_{i}\right)$ and the velocity vector $\mathbf{v}_{i}$.

After substitution of the current density in Eq. (3), the excitation equation can be rewritten in the form

$$
\begin{aligned}
\frac{d}{d z} C_{ \pm q}(z, f)= & \pm \frac{1}{\mathcal{N}_{q}(f)} e^{ \pm j k_{z q} z} \sum_{i} Q_{i} e^{-j 2 \pi f t_{i}(z)} \\
& \times\left\{\frac{\mathbf{v}_{\perp_{i}}}{v_{z_{i}}} \cdot \tilde{\mathcal{E}}_{ \pm q_{\perp}}^{*}\left(x_{i}, y_{i}\right)+\tilde{\mathcal{E}}_{ \pm q_{z}}^{*}\left(x_{i}, y_{i}\right)\right\}
\end{aligned}
$$

The velocity of each particle, in the presence of an electric field $\mathbf{E}(\mathbf{r}, t)$ and a magnetic field $\mathbf{B}(\mathbf{r}, t)=$ $\mu \mathbf{H}(\mathbf{r}, t)$, is found from the Lorentz force equation:

$$
\frac{d}{d t}\left(\gamma_{i} \mathbf{v}_{i}\right)=-\frac{e}{m}\left[\mathbf{E}\left(\mathbf{r}_{i}, t\right)+\mathbf{v}_{i} \times \mathbf{B}\left(\mathbf{r}_{i}, t\right)\right],
$$

where $e$ and $m$ are the electron charge and mass, respectively. The fields represent the total (DC and AC) forces operating on the particle, and include the self-field due to space-charge. The Lorentz relativistic factor $\gamma_{i}$ of each particle is found from the equation for kinetic energy:

$$
\frac{d \gamma_{i}}{d t}=-\frac{e}{m c^{2}} \mathbf{v}_{i} \cdot \mathbf{E}\left(\mathbf{r}_{i}, t\right)
$$

where $c$ is the velocity of light.

Equations (7) and (8) are arranged by replacing the time derivative $\frac{d}{d t}=v_{z_{i}} \frac{d}{d z}$ such that the coordinate of the propagation axis $z$ becomes the independent variable. This defines a transformation of variables for each particle, which enables one to formulate the three-dimensional equations of motion in terms of $z$ :

$$
\frac{d \mathbf{v}_{i}}{d z}=\frac{1}{\gamma_{i}}\left\{-\frac{e}{m} \frac{1}{v_{z_{i}}}\left[\mathbf{E}\left(\mathbf{r}_{i}, t\right)+\mathbf{v}_{i} \times \mathbf{B}\left(\mathbf{r}_{i}, t\right)\right]-\mathbf{v}_{i} \frac{d \gamma_{i}}{d z}\right\}
$$

$$
\frac{d \gamma_{i}}{d z}=-\frac{e}{m c^{2}} \frac{1}{v_{z_{i}}} \mathbf{v}_{i} \cdot \mathbf{E}
$$

The time $t_{i}(z)$ is a function of the time $t_{0 i}$ when a particle entered at $z=0$, and of the instantaneous longitudinal velocity $v_{z i}(z)$ :

$$
t_{i}(z)=t_{0_{i}}+\int_{0}^{z} \frac{1}{v_{z_{i}}\left(z^{\prime}\right)} d z^{\prime}
$$

Equations (2), (6), and (9)-(11) form a closed set of nonlinear equations, which enable a self-consistent calculation of both the radiated field and the beam trajectory. Errors arising in numerical integration of the equations may be controlled by checking the energy balance,

$$
W_{\mathrm{EM}}(z)+W_{\text {beam }}(z)=W_{\mathrm{EM}}(0)+W_{\text {beam }}(0),
$$

at each point $z$ of the beam trajectory, where

$$
W_{\mathrm{EM}}(z)=\int_{0}^{\infty} \frac{d W(z)}{d f} d f
$$

is the total energy flux of the emitted electromagnetic field defined by integration of the energy flux spectral density given by 


$$
\begin{aligned}
\frac{d W(z)}{d f} & =\frac{1}{2} \operatorname{Re}\left\{\iint\left[\tilde{\mathbf{E}}(\mathbf{r}, f) \times \tilde{\mathbf{H}}^{*}(\mathbf{r}, f)\right] \cdot \hat{\mathbf{z}} d x d y\right\} \\
& =\frac{1}{2} \sum_{q}\left[\left|C_{+q}(z, f)\right|^{2}-\left|C_{-q}(z, f)\right|^{2}\right] \operatorname{Re}\left\{\mathcal{N}_{q}(f)\right\}
\end{aligned}
$$

and

$$
W_{\text {beam }}(z)=\frac{m_{0} c^{2}}{e} \sum_{i}\left|q_{i}\right|\left[\gamma_{i}(z)-1\right]
$$

is the beam energy $\left[\gamma_{i}(z)\right.$ is the Lorentz relativistic factor of the particle $i$ at the point $z$ ].

\section{ANALYTICAL EVALUATION}

Neglecting the influence of the emitted electromagnetic field on the radiating electrons, the mode expansion coefficients $\tilde{C}_{q}(z, f)$ of the undulator synchrotron radiation field emitted by a single wiggling particle is found in analytical form [19]:

$$
\tilde{C}_{q}\left(z=L_{w}, f\right)=\mathcal{A}_{q} \operatorname{sinc}\left(\frac{1}{2} \theta_{q} L_{w}\right) e^{j\left[w t_{0}+(1 / 2) \theta_{q} L_{w}\right]},
$$

where $\mathcal{A}_{q}$ stands for a normalization coefficient, $\operatorname{sinc}(\alpha) \equiv \sin (\alpha) / \alpha, t_{0}$ is the time when the electron entered into the interaction area, $L_{w}$ is the length of the interaction area, and

$$
\theta_{q} \equiv \frac{w}{v_{z}}-\left(k_{z q}+k_{w}\right)
$$

is the detuning parameter ( $k_{w}$ is the wiggler wave number).

In the low-gain regime, the electromagnetic field emitted by a bunch of $N_{e}$ electrons is given in the terms of the sum of radiation of single particles (16) over all the electrons in the bunch:

$$
\tilde{C}_{q}\left(z=L_{w}, f\right)=\mathcal{A}_{q} \sum_{i=1}^{N_{e}} \operatorname{sinc}\left(\frac{1}{2} \theta_{q_{i}} L_{w}\right) e^{j\left[w t_{0_{i}}+(1 / 2) \theta_{q_{i}} L_{w}\right]}
$$

The coefficient $\mathcal{A}_{q}$ is assumed to be approximately constant for all the electrons in the bunch. A coherent summation in (18) may be obtained, if at a synchronism frequency $f_{s}$ the condition $\operatorname{sinc}\left(\frac{1}{2} \theta_{q_{i}} L_{w}\right) \approx 1$, or $\theta_{q_{i}} \approx 0$ takes place, and the phase-matching condition $w_{s} t_{0_{i}}+\frac{1}{2} \theta_{q_{i}} L_{w} \approx 0$ is satisfied for all the electrons. This situation results in SR emission of a single ultrashort $\left(T_{b} \ll 1 / f_{s}\right)$ bunch, when the electrons radiate almost in the same phase. The radiated field is proportional to the number $N_{e}$ of electrons in the bunch (to the total charge of the drive bunch). The energy flux spectral density of the emitted radiation is then given by

$$
\frac{d W}{d f}=\frac{1}{2}\left|\tilde{C}_{q}\left(L_{w}, f\right)\right|^{2} \operatorname{Re}\left\{\mathcal{N}_{q}(f)\right\} \approx N_{e}^{2} \frac{\mathcal{A}_{q}^{2}}{2} \operatorname{Re}\left\{\mathcal{N}_{q}(f)\right\}
$$

As described above, the emission from different electrons becomes incoherent in phase causing destructive interference if the bunch duration is increased to be close or above the period $1 / f_{s}$ of the emitted radiation. This destructive interference can, however, be reduced and the sum in (18) may remain partially coherent in the vicinity of the synchronism frequency $f_{s}$, if (a) most of the sinc functions in (18) are positive: $\operatorname{sinc}\left(\frac{1}{2} \theta_{q_{i}} L_{w}\right)>0$; and (b) the phase-matching condition $w_{s} t_{0_{i}}+\frac{1}{2} \theta_{q_{i}} L_{w} \approx 0$ is realized. The first condition can be expressed in the form

$$
\left|\frac{w_{s}}{v_{z_{i}}}-\left(k_{z_{i}}+k_{w}\right)\right| \lesssim \frac{2 \pi}{L_{w}},
$$

and by introducing

$$
k_{z_{q}}+k_{w} \equiv \frac{w_{s}}{\bar{\beta}_{z} c}
$$

it can be rewritten as

$$
\left|\frac{1}{\beta_{z_{i}}}-\frac{1}{\bar{\beta}_{z}}\right| \lesssim \frac{\lambda_{s}}{L_{w}},
$$

where $\bar{\beta}_{z} c$ is the mean longitudinal velocity of the bunch in the $z$-direction, $\lambda_{s}$ is the radiation wavelength, and $\beta_{z_{i}}=$ $v_{z_{i}} / c$.

The phase-matching condition can be satisfied only if the drive electron bunch is premodulated and the longitudinal velocities of the electrons are distributed as follows:

$$
\beta_{z}(t)=\bar{\beta}_{z} \frac{L_{w}}{L_{w}-2 \bar{\beta}_{z} c t}
$$

assuming $\beta_{z}(t=0)=\bar{\beta}_{z}$.

Substitution of (22) into (21) provides the condition of the maximal bunch duration:

$$
|t| \lesssim \frac{1}{2 f_{s}}
$$

showing that enhancement of SR emission may be expected also for bunches of $T_{b} \sim 1 / f_{s}$.

From Eq. (22), the energy distribution along the drive electron bunch is derived to be

$$
E_{k}(t)=m_{0} c^{2}\left\{\sqrt{\frac{1+a_{w}^{2}}{1-\bar{\beta}_{z}^{2} /\left(1-2 \bar{\beta}_{z} c t / L_{w}\right)^{2}}}-1\right\},
$$

where $a_{w}=e B_{w} /\left(k_{w} m c\right)$ is the wiggler parameter. Noting that

$$
\left(\frac{2 \bar{\beta}_{z} c}{L_{w}}\right)|t| \lesssim\left(\frac{2 \bar{\beta}_{z} c}{L_{w}}\right) \frac{1}{2 f_{s}}=\bar{\beta}_{z} \frac{\lambda_{s}}{L_{w}} \ll 1,
$$

a linear approximation of the distribution (24) can be carried out via first order Taylor expansion 


$$
E_{k}(t) \approx E_{k}(t=0)+\left.\left(\frac{d E_{k}}{d t}\right)\right|_{t=0} \cdot t
$$

where

$$
E_{k}(t=0) \equiv m c^{2}\left\{\sqrt{\frac{1+a_{w}^{2}}{1-\bar{\beta}_{z}^{2}}}-1\right\}
$$

and the rate of modulation is

$$
\left.\left(\frac{d E_{k}}{d t}\right)\right|_{t=0} \approx m c^{2}\left(\frac{2 \bar{\gamma} \bar{\beta}_{z}^{3}}{1-\bar{\beta}_{z}^{2}}\right) \frac{c}{L_{w}} .
$$

Here

$$
\bar{\gamma}=\frac{m c^{2}+E_{k}(t=0)}{m c^{2}}=\sqrt{1-\frac{1+\bar{a}_{w}^{2}}{\bar{\beta}_{z}^{2}}} .
$$

With energy modulation as given by Eq. (24) or Eq. (25), the SR emission from an electron bunch is considerably enhanced even if its temporal duration is comparable with the radiation period (SR emission of an unmodulated bunch is expected to be strongly suppressed). Energy

TABLE I. Operational parameters for THz FEL.

\section{Accelerator}

Electron beam energy:

Wiggler

Magnetic induction:

Period:

Number of periods:

Waveguide

Rectangular waveguide:

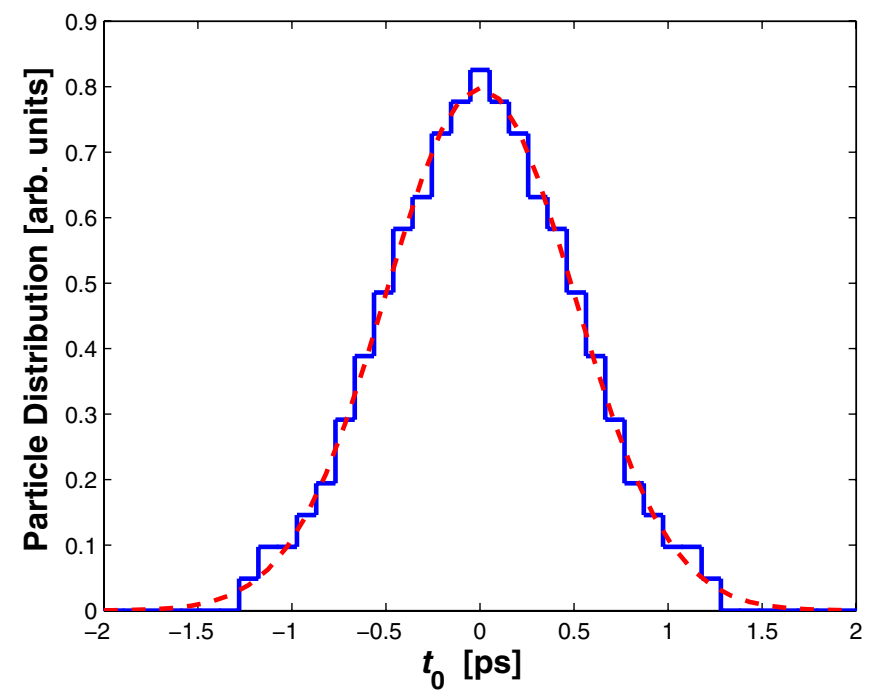

FIG. 2. (Color) Initial time distribution of $N=201$ charged particles in the simulations (bunch duration is $T_{b}=1 \mathrm{ps}$ ). The dashed line shows the respective Gaussian distribution function (30). modulation of the beam reduces the spectral purity of the emitted radiation as compared to SR emission of a "cold" unmodulated ultrashort duration electron bunch. The synchronism frequencies of undulator synchrotron radiation emitted by various electrons differ due to dispersion of their kinetic energies causing broadening of the spectrum. Therefore, the bandwidth of radiation emitted by an energy-modulated bunch includes a supplementary term $\Delta f \approx\left|f_{s}\left(+T_{b} / 2\right)-f_{s}\left(-T_{b} / 2\right)\right|$, in addition to the fundamental bandwidth $f_{s} / N_{w}$ of SR emission ( $N_{w}$ is the number of periods of the wiggler), resulting in a bandwidth

$$
\mathrm{BW} \approx \frac{f_{s}}{N_{w}}+\underbrace{\frac{d f_{s}}{d E_{k}}\left(\frac{d E_{k}}{d t}\right) T_{b}}_{\Delta f},
$$

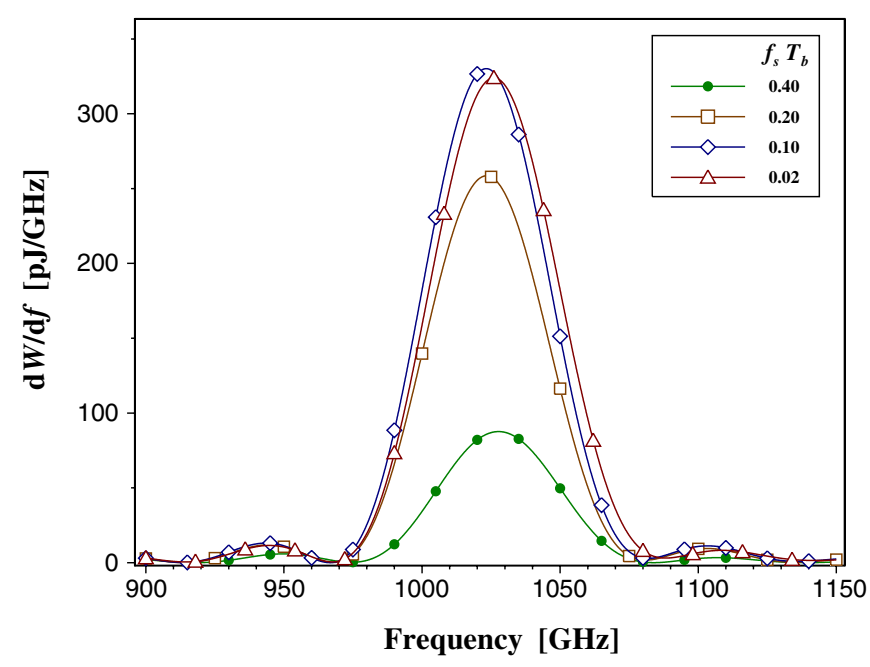

FIG. 3. (Color) Spectrum of radiation emitted by unmodulated $Q=10 \mathrm{pC}$ electron bunches.

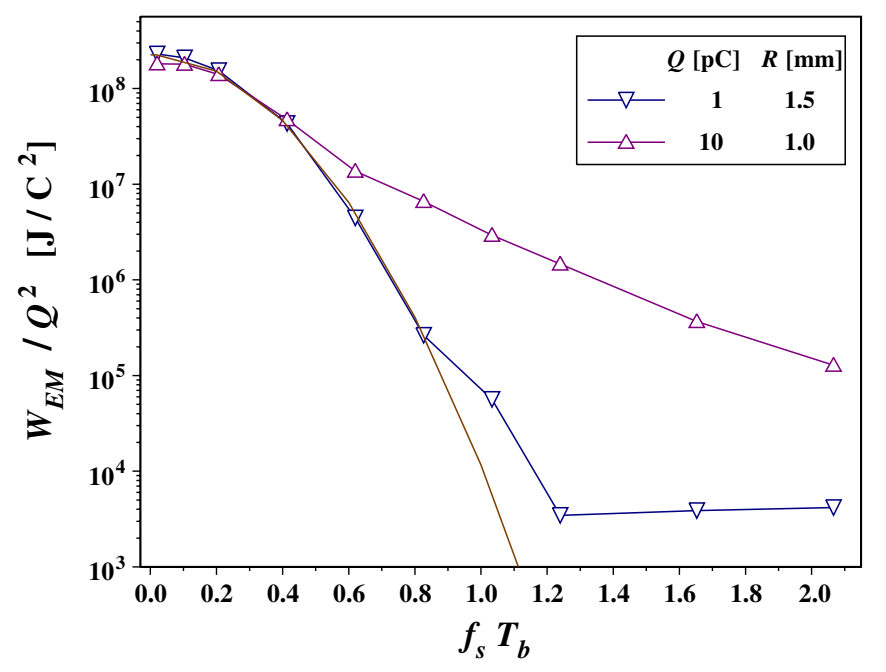

FIG. 4. (Color) Normalized total energy flux $W_{\mathrm{EM}} / Q^{2}$ of superradiant emission emitted by a short electron bunch, as a function of $f_{s} T_{b}$. 

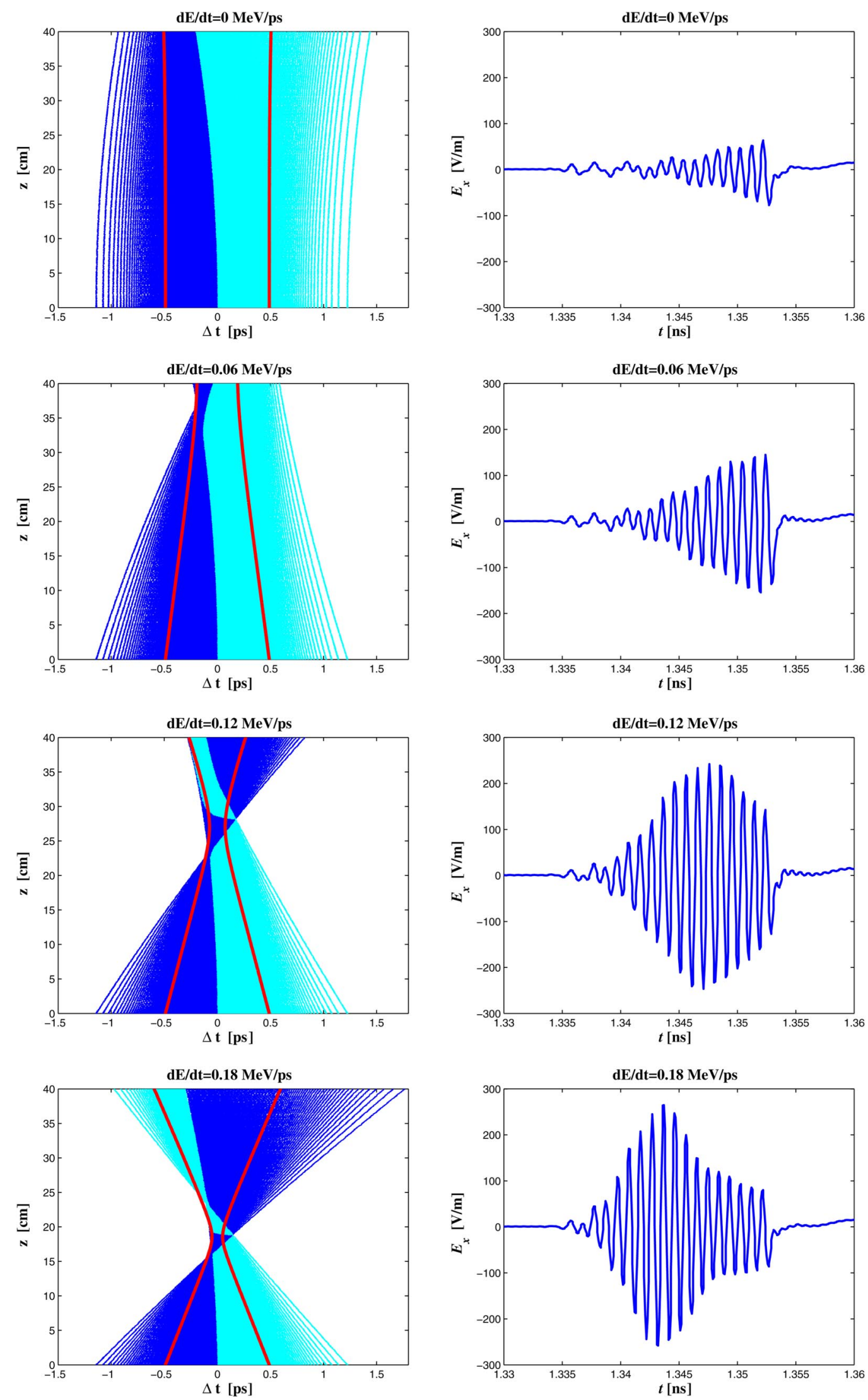

FIG. 5. (Color) Trajectories of electrons relative to the bunch center (left) and emitted time-domain electromagnetic field (right) for different energy modulation rates $d E_{k} / d t$. 
which reveals a linear growth of bandwidth with energy chirp rate $d E_{k} / d t$.

\section{NUMERICAL SIMULATIONS}

To demonstrate the effect of beam energy modulation on the FEL super-radiance, numerical simulations with the WB3D code [25] were carried out. Operational parameters for a terahertz regime free-electron laser considered in the simulations are given in the Table I. In the simulations, $N=201$ charged particles are taken with an initial temporal distribution having a Gaussian shape:

$$
\sqrt{\frac{2}{\pi}} \frac{1}{T_{b}} e^{-2 t^{2} / T_{b}^{2}}
$$

$T_{b}$ is the duration of the electron bunches. A typical example of the distribution for a bunch with $T_{b}=1.0 \mathrm{ps}$ is shown in Fig. 2.

Figure 3 presents the energy spectral density of superradiant emission from unmodulated electron bunches with a total charge of $Q=10 \mathrm{pC}$. It shows that extremely short electron bunches ( $T_{b} \lesssim 0.2 \mathrm{ps}$ ) have to be used in order to generate strong super-radiant emission. A total energy flux of $W_{\mathrm{EM}} \approx 18 \mathrm{~nJ}$ is obtained with such ultrashort bunches which becomes drastically smaller if longer bunches are used. This is demonstrated clearly in Fig. 4, where the normalized total energy flux $W_{\mathrm{EM}} / Q^{2}$ of the emitted radiation is shown as a function of the relative duration $f_{s} T_{b}$ of the radiating electron bunch. According to (19), the normalized total energy flux is not sensitive to the beam total charge $Q$ if super-radiant emission from ultrashort bunches takes place.

Space-charge effects are expected to be dominant and to play an important role in the interaction if high charge density bunches are used. In order to evaluate the effect of space-charge, simulations with low charge density (total charge $Q=1 \mathrm{pC}$, beam radius $R_{b}=1.5 \mathrm{~mm}$ ) were carried out. Inspection of Fig. 4 for the ultrashort pulse regime $\left(f_{s} T_{b} \rightarrow 0\right)$ reveals a small difference in the resulting normalized SR intensity, showing negligible space-charge effects for our operational parameters.

If duration of an unmodulated bunch approaches the radiation period or exceeds it, $f_{s} T_{b} \geqslant 1$, spontaneous emission takes place; it is much lower in comparison to SR emission of ultrashort electron bunches. However, even in this case linear energy modulation (25) of electrons in the bunch enables one to enhance SR emission considerably. The energy modulation gives rise to a density compression of the emitting bunch within the interaction area. This effect is demonstrated by the electron trajectories shown in Fig. 5 (left side). Without energy modulation (chirp rate is $d E_{k} / d t=0$ ), particles traverse the undulator without changing significantly their initial temporal distribution. Energy modulation results in a temporal selfcompression, during a certain time period the bunch be-

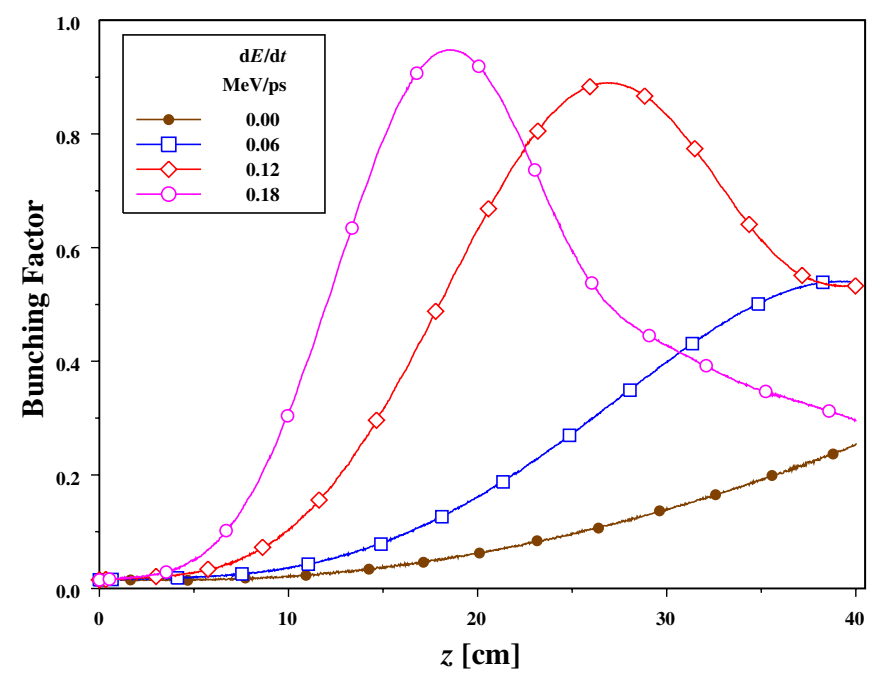

FIG. 6. (Color) Typical variations of bunching factor (31) along the interaction region. The simulations were carried out with $Q=10 \mathrm{pC}$ and $f_{s} T_{b} \approx 1$.

comes an "ultrashort" one, satisfying the conditions for enhanced super-radiant emission. In this short period the most intensive radiation is emitted, as seen from the emitted electric field in the time-domain shown in Fig. 5 (right side). The effect of energy modulation on density compression can be evaluated through the bunching factor

$$
b(z)=\frac{1}{N} \sum_{i=1}^{N} e^{j w_{s} t_{i}(z)}
$$

This quantity is given in Fig. 6 for a several values of the energy chirp rate $d E_{k} / d t$.

The total energy flux $W_{\mathrm{EM}}$ of SR emission as a function of the energy chirp rate $d E_{k} / d t$ is shown in Fig. 7. One can note that energy modulation increases the intensity of the emitted radiation by a several orders of magnitude.

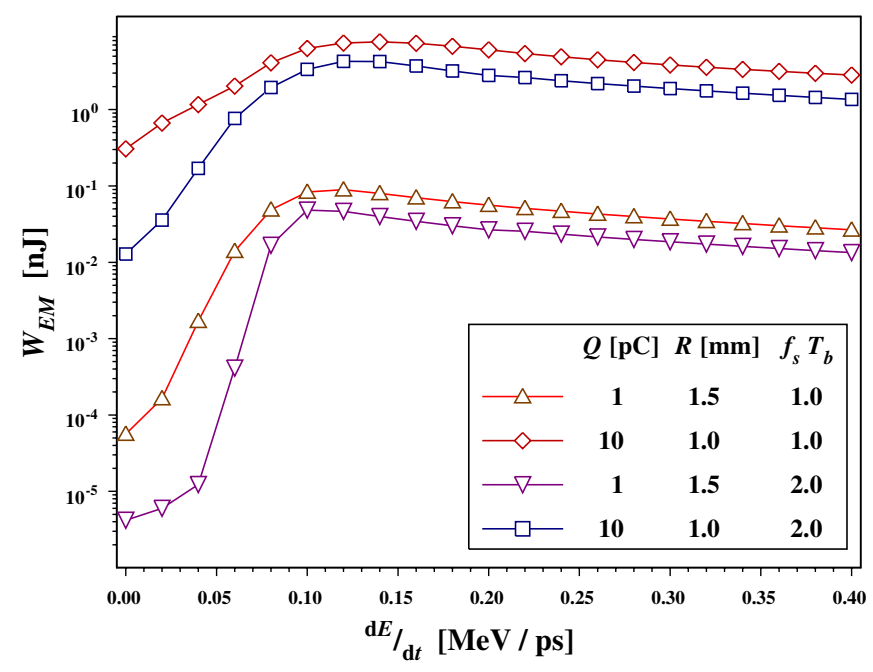

FIG. 7. (Color) The total emitted energy flux as function of energy chirp rate $d E_{k} / d t$. 


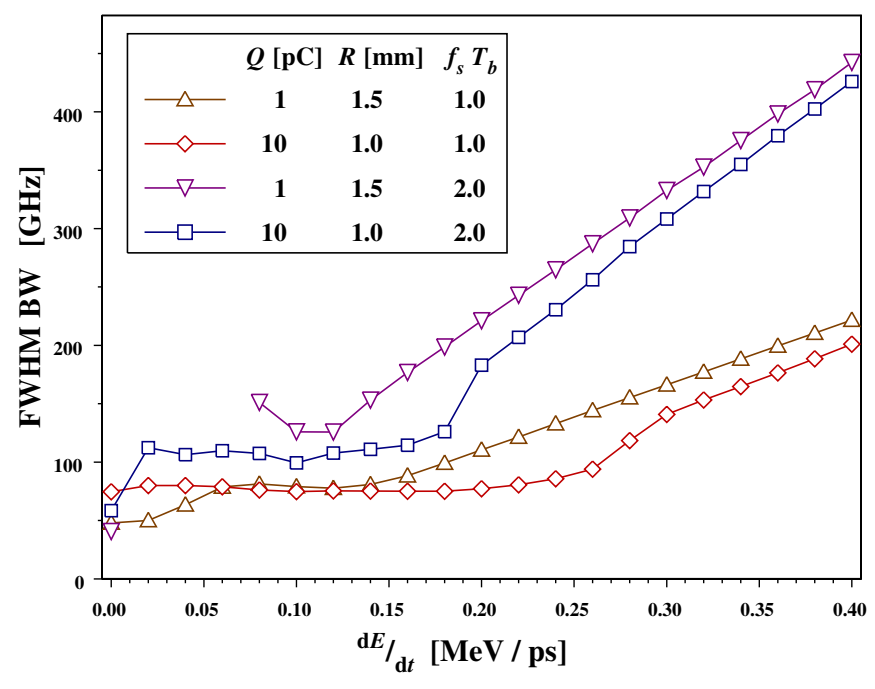

FIG. 8. (Color) FWHM bandwidth of the radiation as a function of the energy chirp rate $d E_{k} / d t$.

Although space-charge forces reduce the effect by decreasing the bunch compression, the SR emission is very strong. The total energy flux reaches saturation at $d E_{k} / d t \approx$ $0.12 \mathrm{MeV} / \mathrm{ps}$, close to the analytical result $d E_{k} / d t \approx$ $0.17 \mathrm{MeV} / \mathrm{ps}$ obtained from Eq. (27). Note that with longer bunches $f_{s} T_{b}>1$, where spontaneous emission mainly is generated when no modulation is introduced, the enhancement of SR emission due to the energy modulation is even stronger.

Full-width half-maximum (FWHM) bandwidth of the emitted radiation line as a function of the energy chirp rate $d E_{k} / d t$ is presented in Fig. 8. As predicted by (29), the radiation bandwidth grows linearly with $d E_{k} / d t$ at high energy chirp rates. However, at medium values of the chirp rate $\left(d E_{k} / d t \approx 0.10 \div 0.14 \mathrm{MeV} / \mathrm{ps}\right)$, the energy flux

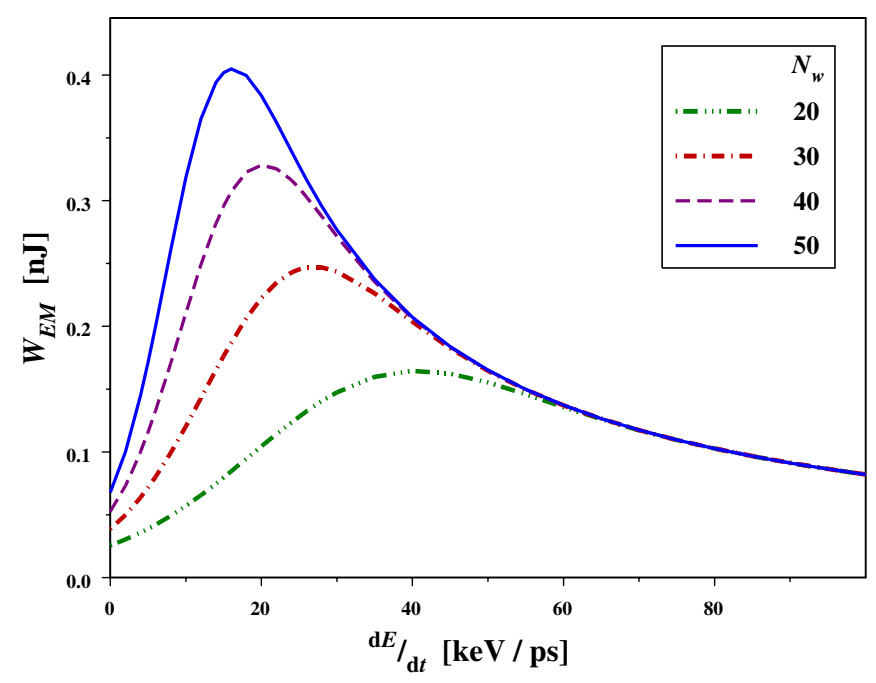

FIG. 9. (Color) Reduction of the energy chirp rate $d E_{k} / d t$ with the wiggler length. The beam energy is $E_{k}=1.8 \mathrm{MeV}$ and the resulted radiation frequency is $f_{s} \approx 475 \mathrm{GHz}$.
$W_{\mathrm{EM}}$ reaches its peak value with bandwidths close to that expected from an ultrashort bunch.

According to (27), enhancement of SR emission can be observed at lower energy chirp rates if a lower beam energy and a longer wiggler are considered. To demonstrate this effect, the beam energy was reduced to $E_{k}=1.8 \mathrm{MeV}$ (this shifts the synchronism frequency down to about $f_{s} \approx$ $475 \mathrm{GHz}$ ). In addition, the total energy flux resulted after $20,30,40$, or 50 wiggler periods was calculated in order to show the dependence on wiggler length. The results of simulations are given in Fig. 9, revealing that with lower beam energy the peak of the total energy flux shifts down to the chirp rate of $d E_{k} / d t \approx 40 \mathrm{keV} / \mathrm{ps}$ (for $N_{w}=20$ ). The reduction is even stronger (down to about $16 \mathrm{keV} / \mathrm{ps}$ ) if a $N_{w}=50$ long wiggler is considered.

\section{CONCLUSION}

In FELs driven by a single short electron bunch or by a train of bunches, super-radiant emission is strongly suppressed at high frequencies (above millimeter wavelengths). Super-radiance can, however, be greatly enhanced by means of energy modulation of the drive beam. The effect was studied using an analytical spacefrequency model and also demonstrated by numerical simulations with the WB3D code. It is shown that the effect is due to density compression of the drive electron bunch inside the interaction area. The enhancement is obtained with relatively low charge bunches having a temporal duration comparable to the radiation period.

According to this theoretical study, energy chirp rates required for enhancement of super-radiant emission in $\mathrm{THz}$ FELs are found to be rather high. Nevertheless, emerging RF-LINAC FEL schemes enable obtaining considerably high energy chirp rates. That can be achieved by using a "phase-matching section" within the FEL beam line [23]. Also note that, according to (27), the chirp rates are remarkably reduced when lower beam energy and longer wiggler are utilized.

The discussed scheme can be considered in the development of a pulsed FEL generating intensive radiation at submillimeter wavelengths (terahertz frequencies) with reasonable spectral quality.

\section{ACKNOWLEDGMENTS}

The authors would like to thank A. L. Eichenbaum for his help and useful remarks.

[1] H. Motz, J. Appl. Phys. 22, 527 (1951).

[2] W. Schottky, Ann. Phys. (Leipzig) 57, 541 (1918).

[3] S. O. Rice, Bell Syst. Tech. J. 23, 282 (1944).

[4] S. O. Rice, Bell Syst. Tech. J. 24, 46 (1945).

[5] Noise in Electron Devices, edited by L. D. Smulin and H. A. Haus, The MIT Press Classics Series (MIT Press, Cambridge, MA, 1959). 
[6] B. Kincaid, J. Appl. Phys. 48, 2684 (1977).

[7] J. M. J. Madey, Nuovo Cimento B 50, 64 (1979).

[8] A. N. Didenko et al., Sov. Phys. JTEP 49, 973 (1979).

[9] N.M. Kroll, Physics of Quantum Electronics: FreeElectron Generators of Coherent Radiation (AddisonWesley, Reading, MA, 1980), Vol. 7.

[10] H. P. Freund et al., Phys. Rev. A 24, 1965 (1981).

[11] W. B. Colson, IEEE J. Quantum Electron. 17, 1417 (1981).

[12] H. A. Haus and M. N. Islam, J. Appl. Phys. 54, 4784 (1983).

[13] K. J. Kim, AIP Conf. Proc. 184, 565 (1989).

[14] F. V. Hartemann, Phys. Rev. E 61, 972 (2000).

[15] S. Y. Cai, J. Cao, and A. Bhattacharjee, Phys. Rev. A 42, 4120 (1990).
[16] N. S. Ginzburg and A. S. Sergeev, Opt. Commun. 91, 140 (1992).

[17] F. Ciocci et al., Phys. Rev. Lett. 70, 928 (1993).

[18] A. Gover et al., Phys. Rev. Lett. 72, 1192 (1994).

[19] Y. Pinhasi and A. Gover, Nucl. Instrum. Methods Phys. Res., Sect. A 393, 343 (1997).

[20] R. H. Dicke, Phys. Rev. 93, 99 (1954).

[21] Y. Pinhasi and Yu. Lurie, Phys. Rev. E 65, 026501 (2002).

[22] A. Gover, Phys. Rev. ST Accel. Beams 8, 030701 (2005).

[23] A. Doria et al., Phys. Rev. Lett. 80, 2841 (1998).

[24] N. S. Ginzburg et al., Opt. Commun. 231, 303 (2004).

[25] Y. Pinhasi, Yu. Lurie, and A. Yahalom, Nucl. Instrum. Methods Phys. Res., Sect. A 475, 147 (2001).

[26] Y. Pinhasi and A. Gover, Phys. Rev. E 51, 2472 (1995). 